\title{
Cosa fatta (e risposta data) capo ha!
}

\author{
Chiara Coluccia
}

PUBBLICATO: 05 MARZO 2019

\begin{abstract}
Quesito:
Molti lettori chiedono spiegazioni sull'espressione proverbiale cosa fatta capo ha: qual è il significato in Dante e nel parlare comune? E si dice cosa fatta capo ha oppure cosa fatta, capo a, nel senso che si va a capo?

\section{Cosa fatta (e risposta data) capo ha!}

La celebre locuzione cosa fatta capo ha (con l'ausiliare ha, non con la preposizione a) è registrata in molti importanti vocabolari. Garzanti 2017 s.v. capo (6.): "ciò che è stato fatto non può più essere cambiato"; SabatiniColetti 2008 s.v. capo (3.): "non si può correggere, modificare l'azione compiuta"; Vocabolario Treccani online: "una cosa fatta non può essere disfatta', cioè riesce al suo capo, al suo effetto"; VOLIT s.v. capo (3.f.): "ciò chè fatto è fatto e non c'è più rimedio", "propriam.: cosa fatta è bell'e finita"; Zingarelli zorg s.v. capo (r.): "ciò che è fatto è fatto e non si può più cambiare". Ricorre in Devoto-Oli 2019 s.v. cosa (3.) senza commento. Tra i lessici storici ed etimologici è registrata in Crusca I e II (con la semplice chiosa 'proverbio'), in Crusca III ('dopo il fatto ogni cosa s'aggiusta'), Crusca IV ('id.'), Crusca V ('id.'), Crusca veronese ('id.'), Tommaseo-Bellini ('id.'), Vocabolario dell'Accademia ('non c'e più rimedio'), GDLI ('cosa fatta non può disfarsi; dopo il fatto se ne trova la ragione [a significare l'irrevocabilità delle cose compiute])', LEI ('ogni azione intrapresa ha uno sbocco [il cui esito non è facile prevedere]; dopo il fatto se ne trova la ragione [a significare l'irrevocabilità delle cose compiutel', per ricordare solo i dizionari piu autorevoli.
\end{abstract}

Saltuariamente è registrata nei repertori correnti di fraseologia, modi di dire, proverbi. Pur con qualche variazione di dettaglio, nel complesso il significato è univoco: sottolinea l'irrevocabilità di un'azione, che non consente recriminazioni o rimedi. Alla lettera significa 'ogni azione [cosa fatta] ha una conclusione [capo]; la parola capo, che indica il punto esatto sia d'inizio (capo d'anno) che di fine (venire a capo di una cosa, concluderla), qui vale 'conclusione, fine'. Ma non è affatto chiaro come i singoli elementi lessicali in sequenza diano luogo al significato metaforico complessivo.

La diffusione nella nostra lingua di tale locuzione (dal valore proverbiale) si collega a un episodio storico preciso, avvenuto a Firenze nei primi mesi del I216. Buondelmonte dei Buondelmonti, cavaliere di una nobile famiglia fiorentina, vien meno all'impegno di sposare una fanciulla degli Amidei, altra importante famiglia cittadina. Gli Amidei si accordano con i Lamberti, con gli Uberti e con altri sodali per decidere la reazione più opportuna. Di fronte a proposte di violenza variabile ai danni dell'autore della violata promessa matrimoniale (offendere pubblicamente?, picchiare?, ferire?, uccidere?), Mosca de' Lamberti, per questo collocato nella Commedia tra i seminatori di scandalo, di scisma e (nel suo caso) di discordie (cerchio VIII, nona bolgia: Inferno XXVIII то3-гіI), suggerisce l'omicidio del fedifrago; e suggella il proprio parere con la frase "cosa fatta capo ha" . Lomicidio avviene a Ponte Vecchio, la mattina di Pasqua (data e luogo fortemente simbolici), proprio nel giorno fissato per le nozze di Buondelmonte con un'avvenente giovane della famiglia Donati. All'agguato mortale seguono decenni di continue lotte intestine, origine della partizione di Firenze in guelfi e ghibellini e delle discordie cittadine, "mal seme per la gente tosca" (Inferno XXVIII ro8).

L'episodio ebbe enorme eco nelle fonti del tempo. Senza contare la testimonianza più celebre, quella di Dante, la descrizione degli eventi e la locuzione collegata (con qualche variazione formale) risultano presenti in quasi venti opere collocabili entro i confini del Trecento, vicine o relativamente vicine al fatto di sangue. Molti sono testi cronachistici, o comunque di carattere latamente storico, fiorentini (in ordine cronologico: la Cronica fiorentina di Anonimo, le cronache di Paolino Pieri, Dino Compagni, Giovanni Villani, Marchionne di Coppo Stefani, il Centiloquio di Antonio Pucci, la Storia fiorentina dei Malispini, il Pecorone di Ser Giovanni), e molti sono commenti danteschi (quelli di Jacopo Alighieri, Jacopo della Lana, l'Ottimo Commento, le Chiose Selmiane, Guglielmo Maramauro, le Chiose del falso Boccaccio, Francesco da Buti e le Chiose Vernon). Soltanto Boccaccio nel Filocolo ricorre alla locuzione svincolandola rispetto all'episodio dell'uccisione di Buondelmonte. 
Il brano decisivo dal punto di vista esegetico e che consente una più soddisfacente interpretazione delle altre fonti, meno esplicite su questo punto, è quello di Paolino Pieri, cronista fiorentino che descrive eventi accaduti nel periodo compreso tra il ro8o e il I205. Nel cap. 55 delle sue Croniche della cittá di Firenze il ragionamento di Mosca Lamberti si svolge secondo una concatenazione stringente: nella situazione di incertezza sul da farsi (su cui insistono molte delle altre fonti elencate), Mosca premette che "cosa fatta capo à" "qualsiasi azione intrapresa ha una conclusione' e fa seguire alla locuzione un avvertimento che segnala l'imprevedibilità delle conseguenze: "ma ttalora non chente vuole né chente crede o disengna" 'l'esito dell'azione può risultare imprevisto e diverso da quello desiderato'; di conseguenza, Mosca afferma risolutamente la necessità della scelta più drastica: "Se voi il fedite sança ucciderlo, voi non ca[n]perete nel mondo d'inançi lì" 'voi non vivrete un momento di più' (a causa della sicura ritorsione che subirete da lui lasciato in vita). Su questa base documentaria possiamo concludere che nell'italiano antico cosa fatta capo ha significa semplicemente 'ogni azione intrapresa ha una conseguenza' (il cui esito non è facile prevedere). Non si trova allusione, nelle prime attestazioni, al senso di irrevocabilità o di irrimediabilità di una scelta né si rinviene l'esortazione ad abbandonare incertezze o esitazioni, presenti nella nostra tradizione lessicografica storica ed etimologica (oltre che nei lessici dell'italiano contemporaneo).

Nei secoli successivi quei fatti e la frase che li suggella vengono ricordati ancora, con accenti vivaci, da Machiavelli, da Guicciardini e da un novelliere come Matteo Bandello.

Progressivamente, la locuzione si slega dall'episodio dell'uccisione di Buondelmonte e assume il carattere sentenzioso e proverbiale che conserva nell'italiano contemporaneo, almeno a partire dall'Amor costante di Alessandro Piccolomini, opera stampata a Venezia nel I540. Dai lessici storici ed etimologici e dalle banche dati si ricavano occorrenze della frase in accezione allusiva e marcatamente sentenziosa in testi di Battista Guarini, Salviati, Sarpi, Botta, Guerrazzi, Gioberti. E inoltre nelle raccolte Delle phrasi toscane di Giovanni Stefano da Montemerlo (1566) e Proverbi italiani e latini raccolti da Francesco Lena (1694), in testi teatrali come Glinganni, commedia di Domenico Cornacchini (I605) e in altre opere.

Come spiegare l'evoluzione semantica che sovrappone (e spesso sostituisce, secondo i repertori elencati prima) al significato letterale il valore proverbiale che leggiamo in questi autori? Con tutta probabilità entra in ballo la drammaticità dell'episodio a cui la frase è stata originariamente associata, episodio al quale Dante e tante altre fonti attribuiscono l'origine stessa delle sanguinose discordie di Firenze.

Con questo valore allusivo e accentuatamente sentenzioso, l'antica locuzione gode nel corso dei secoli di fortuna non esigua. È interessante l'adozione che nei primi decenni del secolo scorso ne fa Gabriele D'Annunzio, con vasta eco nella retorica fascista. La frase (allettante anche per il richiamo implicito a Dante e alla tradizione letteraria) viene usata da D'Annunzio per sancire l'irrevocabilità dell'impresa fiumana, divenuta leggendaria in pochi giorni, e per annunciare, il I2 settembre 1920, che avrebbe inviato al Senato americano la nuova delibera del Consiglio di Fiume contro il Patto di Londra: "Con un taglio nettissimo noi abbiamo reciso il nodo che nessuno potrà mai riannodare. Cosa fatta capo ha" (è usata anche in Merope, Canzone di Dardanelli, II2-II4, a. I9II-I2, questa volta con più canonico riferimento a Mosca Lamberti e al celebre episodio storico). Al motto si accompagnano dei disegni, voluti dallo stesso poeta e realizzati da Adolfo De Carolis, in cui compaiono elementi iconici fortemente simbolici: il nodo reciso da un pugnale rappresenta il nodo scorsoio messo dal presidente Wilson intorno alla gola dell'Italia, stabilendo umilianti condizioni di pace (e in questo caso capo potrebbe alludere anche alla qualifica di 'comandante' identificativa del poeta) e i pugnali neri alludono a quelli sguainati dai legionari durante il giuramento di fedeltà.

Ad un ambito storico e culturale non molto dissimile appartiene la raccolta di "cantate" L'Arcitaliano (I928) di Curzio Malaparte, in cui si rinvengono versi come questi: "o Italiani ammazzavivi | il bel tempo torna già: | tutti i giorni son festivi $\mid$ se vendetta si farà $\mid$ son finiti i tempi cattivi $\mid$ chi ha tradito pagherà. | Pace ai morti e botte ai vivi: | cosa fatta capo ha.| Spunta il sole e canta il gallo, | o Mussolini, monta a cavallo".

Dal controllo incrociato del GDLI e della BIZ risulta un altro esempio letterario novecentesco, in Federigo Tozzi, Giovani. La casa venduta (1920): "- Ormai, se Dio vuole, cosa fatta capo ha -" (la frase viene pronunziata dall'acquirente di una abitazione di fronte a riaffioranti resipiscenze e incertezze del venditore).

Ai nostri giorni le incertezze dei lettori da cui ha preso spunto questa nota troverebbero conferma in un dialogo del romanzo Ho voglia di te di Federico Moccia (uscito nel 2010), p. 277 (il grassetto è mio): «Beh, meglio cosi. 
Cosa fatta, capo ha." "Che vuol dire?" "Be', si dice." "Mi rigiro nel letto e infilo la testa sotto uno dei cuscini. Mio fratello. Dice cose che non sa neanche cosa vogliono dire"».

Ma in direzione contraria vanno, in modo decisivo, altre indicazioni. La locuzione ricorre per due volte, a distanza di due mesi esatti, nelle dichiarazioni del vicepresidente del Consiglio Matteo Salvini, politico al vertice di un partito d'ispirazione populista, che fa spesso ricorso a un linguaggio aggressivo e diretto, intenzionalmente rivolto a un pubblico lontano da qualsiasi complessità di natura lessicale o semantica. Ecco i dati: giovedì i8 ottobre 20I8, annunciando l'intenzione di non partecipare al Consiglio dei Ministri del giorno dopo, Salvini dichiara: "Cosa fatta capo ha. Io quando prendo impegni con Di Maio e i cittadini li mantengo". E ancora, il I2 dicembre 20I8, a proposito della legge di bilancio: "Stiamo facendo il possibile e l'impossibile perché entro la fine dell'anno, come da tempi previsti, ci sia finalmente una manovra economica che abbiamo elaborato con mille sforzi, ragionevolezza e buon senso. Se da Bruxelles arriverà altrettanto buon senso, cosa fatta capo ha, ci occuperemo di altro".

Inoltre attestazioni molto recenti (tutte del 2018) si registrano anche in ambiti diversi, ma altrettanto popolari, come gli articoli giornalistici di argomento sportivo: tennis (a proposito della nuova formula della Coppa Davis: "è inutile oggi ipotizzare e speculare sulla correttezza dei votanti, sulla potenza dei soldi e delle Tv [...], etcetera etcetera. Cosa fatta capo ha. Basta recriminare, insinuare, lamentarsi"); equitazione (sull'abolizione della finale a quattro: "la finale a quattro rappresentava un momento di spettacolo [...] assolutamente unico nel suo genere, e che si poteva ammirare solo una volta ogni quattro anni. Ma ormai cosa fatta capo ha"); ciclismo («il corridore della Sky non ha violato alcuna norma antidoping. "Un solo campione di urina non è un indicatore affidabile della quantità inalata", la spiegazione del tribunale dell'Uci. Cosa fatta, capo ha»); basket ("tutto, al momento, si colloca oltre i confini del grottesco. Un altro aggettivo non mi viene. Cosa fatta, capo ha"); calcio ("Cosa fatta, capo ha. Ma le conseguenze sono imprevedibili... Il Paris Saint-Germain dovrà fare a meno di Neymar per la partita dell'anno").

In conclusione, nell'italiano contemporaneo la locuzione gode di buona salute ed è usata comunemente, con sfumature di significato differenti da quelle che aveva al momento della sua comparsa.

\section{Nota bibliografica:}

- Chiara Coluccia, Cosa fatta capo ha. Origine e storia di una locuzione, in "Lingua Nostra", LXV, 2004, pp. 7382.

- Paolino Pieri, Croniche della cittá di Firenze, a cura di Chiara Coluccia, Lecce - Rovato (BS), Pensa MultiMedia Editore, 2015 seconda ed.

- Reale Accademia d'Italia, Vocabolario della lingua italiana, Milano, Società Anonima per la pubblicazione del Vocabolario della lingua italiana, I94I.

- Vocabolario degli Accademici della Crusca Oltre le giunte fatteci finora, cresciuto d'assai migliaia di voci e modi de' Classici, le piu trovate da' Veronesi, dedicato a Sua Altezza Imperiale il principe Eugenio Vice-Re d'Italia, 7 voll., Verona, Dalla stamperia di Dionigi Ramanzini, I806-II.

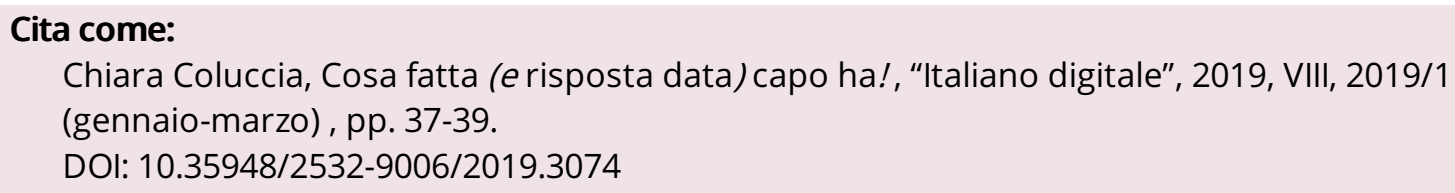

